\title{
Special Session 3 Poster Abstracts
}

\author{
Nicholas A. Walton ${ }^{1}$, Andrew Lawrence ${ }^{2}$ and Roy Williams ${ }^{3}$ (eds.) \\ ${ }^{1}$ Institute of Astronomy, University of Cambridge, Madingley Road, Cambridge CB3 0HA, UK \\ email: naw@ast.cam.ac.uk \\ ${ }^{2}$ University of Edinburgh, Royal Observatory, Blackford Hill, Edinburgh EH9 3HJ, UK \\ email: al@roe.ac.uk \\ ${ }^{3}$ Center for Advanced Computer Research, California Institute of Technology, \\ MC 158-79, 1200 E. California Boulevard, Pasadena, CA 91125, USA \\ email: roy@cacr.caltech.edu
}

\section{An all-sky 2MASS mosaic constructed on the TeraGrid: processing steps for generation of a 20-terabyte 2MASS all-sky mosaic}

\author{
G.B. Berriman ${ }^{1}$, J.C. Good ${ }^{1}$, A.C. Laity ${ }^{1}$, D.S. Katz ${ }^{2}$, J.C. Jacob ${ }^{2}$, L. Brieger ${ }^{3}$, \\ R.W. Moore $^{3}$, R. Williams ${ }^{4}$, E. Deelman ${ }^{5}$, G. Singh ${ }^{5}$ and M.-H. Su \\ ${ }^{1}$ Infrared Processing and Analysis Center, Caltech, Pasadena, CA 91125, USA \\ ${ }^{2}$ Jet Propulsion Laboratory, Pasadena, CA 91109, USA \\ ${ }^{3}$ San Diego Supercomputer Center, La Jolla, CA 92093, USA \\ ${ }^{4}$ Division of Physics, Mathematics and Astronomy, Caltech, Pasadena, CA 91125, USA \\ ${ }^{5}$ Information Sciences Institute, Marina del Rey, CA 90292, USA
}

\begin{abstract}
The Montage mosaic engine supplies on-request image mosaic services for the NVO astronomical community. A companion paper describes scientific applications of Montage. This paper describes one application in detail: the generation at SDSC of a mosaic of the 2MASS All-sky Image Atlas on the NSF TeraGrid. The goals of the project are: to provide a "valueadded' 2MASS product that combines overlapping images to improve sensitivity; to demonstrate applicability of computing at-scale to astronomical missions and surveys, especially projects such as LSST; and to demonstrate the utility of the NVO Hyperatlas format. The numerical processing of an 8-TB 32-bit survey to produce a 64-bit 20-TB output atlas presented multiple scalability and operational challenges. An MPI Python module, MYMPI, was used to manage the alternately sequential and parallel steps of the Montage process. This allowed us to parallelize all steps of the mosaic process: that of many, sequential steps executing simultaneously for independent mosaics and that of a single MPI parallel job executing on many CPUs for a single mosaic. The Storage Resource Broker (SRB) developed at SDSC has been used to archive the output results in the Hyperatlas. The 2MASS mosaics are now being assessed for scientific quality. The input images consist of 4,121,440 files, each $2 \mathrm{MB}$ in size. The input files that fall on mosaic boundaries are opened, read, and used multiple times in the processing of adjacent mosaics, so that a total of 14 TB in 6,275,494 files are actually opened and read in the creation of mosaics across the entire survey. Around 130,000 CPU-hours were used to complete the mosaics. The output consists of 1734 6-degree plates for each of 3 bands. Each of the 5202 mosaics is roughly $4 \mathrm{~GB}$ in size, and each has been tiled into a $12 \times 12$ array of $26-\mathrm{MB}$ files for ease of handling. The total size is about $20 \mathrm{~TB}$ in 750000 tiles.
\end{abstract}

\section{Aladin, a portal for the Virtual Observatory}

\section{T. Boch, P. Fernique, F. Bonnarel, M.G. Allen, O. Bienaymé and S. Derrière CDS, Observatoire de Strasbourg, 11 rue de l'Université, F-67000 Strasbourg, France email: boch@astro.u-strasbg.fr}

Abstract. Created nine years ago, the Aladin sky atlas tool has become a widely-used VO (Virtual Observatory) portal capable of locating data of interest, accessing and exploring distributing 
datasets, and visualizing multi-wavelength data. Its compliance with existing or emerging VO standards, interconnection with other visualization or analysis tools, and its ability to easily compare heterogeneous data are key features that make Aladin a powerful data exploration and integration tool as well as a science enabler. Latest developments include simultaneous access and query to data servers discovered via the IVOA registry, overlays of user-defined instrument fields of view, access to solar system database (SkyBOT) in collaboration with IMCCE, extraction of postage stamps around regions of interest, (re)calibration of images/catalogues and compatibility with the PLASTIC (Platform for AStronomical Tools InterCommunication) protocol to facilitate easy connexion between VO tools. Some developments have been performed in the frame of the european $V O$-Tech project.

The Aladin software is available from the website <http://aladin.u-strasbg.fr/>

\title{
BRAVO (Brazilian Astrophysical Virtual Observatory): data mining development

\author{
R.R.de Carvalho, H.V. Capelato and H.C. Velho \\ INPE, São José dos Campos, Brazil \\ email: reinaldo@das.inpe.br
}

\begin{abstract}
The primary goal of the BRAVO project is to generate investment in information technology, with particular emphasis on datamining and statistical analysis. From a scientific standpoint, the participants assembled to date are engaged in several scientific projects in various fields of cosmology, astrophysics, and data analysis, with significant contributions from international partners. These scientists conduct research on clusters of galaxies, small groups of galaxies, elliptical galaxies, population synthesis, N-body simulations, and a variety of studies in stellar astrophysics. One of the main aspects of this project is the incorporation of these disparate areas of astrophysical research within the context of the coherent development of database technology.Observational cosmology is one of the branches of science experiencing the largest growth in the past few decades. large photometric and spectroscopic surveys have been carried out in both hemispheres. As a result, an extraordinary amount of data in all portions of the electromagnetic spectrum exists, but without standard techniques for storage and distribution. This project will utilize several specific astronomical databases, created to store data generated by several instruments (including SOAR, Gemini, BDA, etc), uniting them within a common framework and with standard interfaces. We are inviting members of the entire Brazilian astronomical community to partake in this effort. This will certainly impact both education and outreach efforts, as well as the future development of astrophysical research. Finally, this project will provide a constant investment in human resources. First, it will do so by stimulating ongoing short technical visits to Johns Hopkins University and Caltech. These will allow us to bring software technology and expertise in datamining back to Brazil. Second, we will organize the Summer School on Software Technology in Astrophysics, which will be designed to ensure that the Brazilian scientific community can take full advantage of the benefits offered by the VO project
\end{abstract}

\section{Status report of Virtual Observatory at the National Central University of China Taipei}

\author{
C.K. Chang ${ }^{1}$, C.M. Ko, ${ }^{2}$ and D. Kinoshita ${ }^{1}$ \\ ${ }^{1}$ Institute of Astronomy, National Central University, Jhongli, China Taipei \\ ${ }^{2}$ Department of Physics and Center for Complex System, National Central University, \\ Jhongli, China Taipei \\ email: rex@astro.ncu.edu.tw
}

Abstract. The idea of virtual observatory (VO) has started to get some attention in Taiwan. However, in order to join the VO community with minimum resources, we identify ourselves as a VO user instead of a developer. We implement the JVO skynode package to create a 2MASS star 
count map. According to level 6 Hierarchical Triangular Mesh (HTM), the sky was divided into 32768 leaves with approximately 1 square degree resolution. We can study Galactic structure and initial mass function with this map. Our next goal is to publish a rather unique light curve data. National Central University is part of Taiwan-America Occultation Survey (TAOS) team. TAOS project will conduct a census of the number of Kuiper belt objects down to a few $\mathrm{km}$ size by monitoring chance stellar occultations by these cometary nuclei. Owing to a special observation strategy, called Zipped mode observation, TAOS can have sub-second photometry. We would like to publish this rather unique light curve database to the VO world.

\title{
Theoretical Virtual Observatory and Grid Web services: VisIVO and new capabilities
}

\author{
A. Costa ${ }^{1}$, U. Becciani ${ }^{1}$, C. Gheller ${ }^{2}$, M. Comparato ${ }^{1}$ and B. Larsson ${ }^{1}$ \\ ${ }^{1}$ INAF OACT, Catania, Italy; ${ }^{2}$ CINECA, Bologna, Italy \\ email: acosta@oact.inaf.it
}

\begin{abstract}
VisIVO is a tool for 3D visualization, it provides an effective and intuitive way of managing, visualizing and analysing the large amount of data produced by observations and numerical simulations. The software is specifically designed to deal with multidimensional data. Catalogues and numerical simulations represent the basic target of VisIVO. The package is written in $\mathrm{C}++$. This poster describes VIsIVO's Grid Web Service (VWS) and its counterpart client side developed in VisIVO. VisIVO's Grid Web Service is developed as a part of the Italian Virtual Observatory, it allows to run applications in grid using the web service technology. The VWS has been designed to work within a Virtual Observatory environment so that the interface for the current application, shown in this poster, and the interfaces for other applications are described by a small and constant piece of WSDL code. Our first application is HOP: an algorithm for finding groups of particles based on the one developed and coded by Daniel Eisenstein \& Piet Hut, Institute for Advanced Study, Princeton, NJ. We have developed VWS using Java AXIS libraries for the server side and C++ AXIS libraries for the client side. The access to the computational resources and storage areas is based on grid services in the INFN Production Grid and from this the VWS inherits asynchronous features, scheduling and matching algorithms. HOP was distributed as RPM Package and was installed in the Worker Node Elements of the INFN Production Grid. The idea is to use external tools through the grid avoiding the integration in our application. We can focus on the I/O management of the jobs and on the standardization of the access methods for the different analysis tools improving the scalability of our solution.
\end{abstract}

\section{SkyMouse, a smart on-line astronomical information collector}

\author{
C. Cui, H. Sun and Y. Zhao \\ National Astronomical Observatories, Chinese Academy of Sciences, Beijing 100012, China \\ email: ccz@bao.ac.cn
}

\begin{abstract}
With the progress of network technologies and astronomical observation technologies, as an example of cyber-infrastructure based sciences, Virtual Observatory is initiated and spreading quickly. More and more on-line accessible database systems and all kinds of services are available. Although astronomers have been aware the importance of 'interoperability', integrated access to the on-line information is still difficult. SkyMouse is a smart system developed by Chinese Virtual Observatory project to let you access different online resource systems easily then ever. Not like some VO efforts on uniformed access systems, for example, NVO DataScope, SkyMouse tries to show a comprehensive overview for a specific object, but not to snatch as much data as possible. Stimulated by a simple 'Mouse Over' on an interested object name, various VO-compliant and traditional databases, i.e., SIMBAD, NED, VizieR, DSS, ADS, are queried by the SkyMouse. An overview for the given object, including basic information, image, observation and references, is displayed in user's default web browser. In the poster, current status and trends of on-line astronomical services are introduced; architecture, topology and
\end{abstract}


key technologies of the SkyMouse system are described; current functions and future plans are listed. The system is accessible at : <http://skymouse.china-vo.org $>$.

\title{
The Virtual Solar-Terrestrial Observatory: interdisciplinary data-driven science

\author{
P.A. Fox ${ }^{1}$, D.L. McGuinness ${ }^{2}$, D. Middleton ${ }^{3}$, L. Cinuini ${ }^{3}$, J. Garcia ${ }^{1}$, P. West ${ }^{1}$, \\ J.A. Darnell ${ }^{1}$ and J. Benedict ${ }^{2}$ \\ ${ }^{1} \mathrm{HAO} / \mathrm{ESSL} / \mathrm{NCAR}$, Boulder, CO, USA \\ ${ }^{2}$ McGuinness Associates, Stanford, CA, USA \\ ${ }^{3} A C / S C D / C I S L / N C A R$, Boulder, CO, USA \\ email: pfox@hao.ucar.edu
}

\begin{abstract}
Virtual Observatories can provide access to vast stores of scientific data: observations and models. As these electronic stores become widely used, there is potential to improve the efficiency, interoperability, collaborative potential, and impact of a wide range of interdisciplinary scientific research. In order to realize this potential, technical challenges need to be addressed concerning (at least) representations and interoperability of data, access, and usability. In the Virtual Solar Terrestrial Observatory (VSTO) project, we are providing an electronic repository of observational data spanning the solar-terrestrial physics domain. We are also implementing semantic web tools and infrastructure for accessing and using the data. Our main contributions include the repository, infrastructure, and tools for the particular solar terrestrial physics as well as the design and infrastructure that may be broadened to cover more diverse science areas and communities of use. In this presentation, we describe the goals, design, current and planned prototypes, and technical infrastructure. We present what we have learned about the processes involved in developing VSTO and the required semantics, how they affect the framework architecture, choice of technologies and service interfaces. VSTO is an NSF-funded joint effort between the High Altitude Observatory and the Scientific Computing Division at the National Center for Atmospheric Research (NCAR) and McGuinness Associates Consulting.
\end{abstract}

\section{Tools and services from the French VO}

\section{F. Genova and the French VO Teams}

CDS, Observatoire Astronomique, UMR CNRS/ULP 7550,

11 rue de l'Université, F-67000 Strasbourg, France

email: genova@astro.u-strasbg.fr

\begin{abstract}
The French VO (Action Spécifique Observatoires Virtuels France - ASOV), a joint action of INSU and CNES, coordinates French participation in the VO for astronomy, solar, heliospheric and space plasma physics, and the study of the planets. It supports teams which develops VO services to uptake VO standards and methods, in particular by organizing tutorials for developers, provides forums for discussion, and funds travel of French participants to IVOA meetings. INSU, on behalf of the French VO, is a member of the IVOA and of Euro-VO, and coordinates the Euro-VO Data Center Alliance, which will be funded by the European Commission as a Coordination Action of the Infrastructure, Communication Network Development program from September 1, 2006. ASOV was created in 2004. Within a few years it has succeeded in creating a national community of VO developers. Several teams participate very actively in the IVOA Interoperability standard development, and collaborate with IT laboratories on VO-related subjects. Most French astronomy laboratories are involved in the development of VO-oriented services and tools, with a wide diversity of actions (observation archives/'science ready' data, value-added data bases and services; tools for visualisation, image analysis, ...; software suites; numerical simulations/theoretical astronomy services, thematic services). A recent census received more than 40 answers describing projects at different scales, some operational, some in development. Details on French VO teams and ASOV actions are available from the French VO Web site: <http://www.france-vo.org/>
\end{abstract}




\title{
Three steps to CIELO
}

\author{
M. Guainazzi and S. Bianchi \\ European Space Astronomy Center, Madrid, Spain \\ email: Matteo.Guainazzi@sciops.esa.int
}

\begin{abstract}
The origin of the soft X-ray emission in obscured Active Galactic Nuclei (AGN) is still largely unknown, despite important progress made possible by recent measurements with Chandra and XMM-Newton. Our understanding of the evolution of accretion onto supermassive black holes, and of its interaction with gas andstars in the dense nuclear environment would receive a dramatic burst by thesolution of this mystery. In this paper, we will: $(a)$ show why high-resolution X-ray spectroscopy is crucial to the solution of this issue; $(b)$ present CIELO, the first catalogue of soft X-ray emission lines in obscured AGN ( $\sim 80$ sources), built from observations of the Reflection Grating Spectrometer (RGS) on-board XMM-Newton; and (c) discuss the implementation of the IVOA Line Data Model in VO tools (such as the SED builder VOSpec), and its application to CIELO. The combination of the unprecedented RGS sensitivity in the soft X-ray regime, and of the VO protocol power leads us to be closer than ever to unveiling the nature of soft X-ray emission in obscured AGN.
\end{abstract}

\section{Graphics interfaces and numerical simulations: Mexican Virtual Solar Observatory}

\author{
L. Hernández ${ }^{1}$, A. González ${ }^{2}$, G. Salas ${ }^{3}$ and A. Santillán ${ }^{3}$ \\ ${ }^{1}$ Instituto de Astronomía, UNAM, Mexico City, DF-04510 Mexico \\ email: liliana@astroscu.unam.mx \\ ${ }^{2}$ Instituto de Ecología, UNAM, Mexico City, DF-04510 Mexico \\ ${ }^{3}$ Cómputo Aplicado-DGSCA, UNAM, Mexico City, DF-04510 Mexico
}

\begin{abstract}
Preliminary results associated to the computational development and creation of the Mexican Virtual Solar Observatory (MVSO) are presented. Basically, the MVSO prototype consists of two parts: the first, related to observations that have been made during the past ten years at the Solar Observation Station (EOS) and at the Carl Sagan Observatory (OCS) of the Universidad de Sonora in Mexico. The second part is associated to the creation and manipulation of a database produced by numerical simulations related to solar phenomena, we are using the MHD ZEUS-3D code. The development of this prototype was made using mysql, apache, java and VSO 1.2. based GNU and 'open source philosophy'. A graphic user interface (GUI) was created in order to make web-based, remote numerical simulations. For this purpose, Mono was used, because it is provides the necessary software to develop and run .NET client and server applications on Linux. Although this project is still under development, we hope to have access, by means of this portal, to other virtual solar observatories and to be able to count on a database created through numerical simulations or, given the case, perform simulations associated to solar phenomena.
\end{abstract}

\section{Integration between solar and space science data for space weather forecast using web services}

\author{
S. Kato \\ Hyogo College of Medicine, Nishinomiya, Japan
}

\begin{abstract}
As the technology develops, the opportunity that the human beings behave in space, and it is still understood that the solar activities (especially the solar flare) influence the airlines communication, the ship communication and the power generator of the electric power company, etc. Forecasting the effects of the solar activities is becoming very important because there is such a background. Our goal is that constructs the detailed model from the Sun to the magnetosphere of the earth and simulates the solar activities and the effects. We try to
\end{abstract}


integrate the existing observational data including the ground observational data and satellite observational data using by web service technology as a base to construct the model. We introduce our activity to combine the solar and space science data in Japan. Methods Generally, it is difficult to develop the virtual common database, but web service makes interconnection among different databases comparatively easy. We try to connect some databases in the portal site. Each different data objects is aggregated to a common data object. We can develop more complex services. We use RELAX NG in order to develop these applications easily. We begin the trial of the interconnection among the solar and space science data in Japan. In the case of solar observational data, we find the activity such as VO, for example, VSO and EGSO, but space science data seems to be very complex. In addition to this, there is time lag that solar activity has an effect on the magnetosphere of the Earth. We discuss these characteristic in the data analysis between the solar and space data. This work was supported by the Grant-in-Aid for Creative Scientific Research 'The Basic Study of Space Weather Prediction' (17GS0208) from the Ministry of Education, Science, Sports, Technology, and Culture of Japan

\title{
Access to high-energy astrophysics through Virtual Observatory: scientific motivation and status of INTEGRAL prototype
}

\author{
P. Kubanek and R. Hudec \\ INTEGRAL Science Data Center, Versoix, Switzerland \\ email:Petr.Kubanek@obs.unige.ch
}

\begin{abstract}
We discuss motivations to create Virtual Observatory access to high-energy data. On example of our INTEGRAL data access we discuss the challenges which we have attacked on creating it, and the current status of the development the VO enabled interface to the public INTEGRAL data.
\end{abstract}

\section{Web Services for public cosmological surveys: the VVDS-CDFS application}

\author{
L. Paioro ${ }^{1}$, B. Garilli ${ }^{1}$, V. Le Brun ${ }^{2}$, P. Franzetti ${ }^{1}$, M. Fumana ${ }^{1}$ and M. Scodeggio ${ }^{1}$ \\ ${ }^{1}$ INAF, IASF Milano 'G. Occhialini', via Bassini 15, I-20133 Milano, Italy \\ email: luigi@lambrate.inaf.it \\ ${ }^{2}$ Laboratoire d'Astrophysique de Marseille, Traverse du Siphon, F-13376 Marseille, France
}

\begin{abstract}
Cosmological surveys (like VVDS, GOODS, DEEP2, COSMOS, etc.) aim at providing a complete census of the universe over a broad redshift range. Often different information are gathered with different instruments (e.g., spectrographs, HST, X-ray telescopes, etc.) and it is only by correctly assembling and easily manipulating such wide sets of data that astronomers can attempt to describe the universe; many different scientific goals can be tackled grouping and filtering the different data sets. When dealing with the huge databases resulting from public cosmological surveys, what is needed is: $(a)$ a versatile system of queries, to allow searches by different parameters (like redshifts, magnitude, colors, etc.) according to the specific scientific goal to be tackled; $(b)$ a cross-matching system to verify or redefine the identification of the sources; and $(c)$ a data products retrieving system to download data related images and spectra. The Virtual Observatory Alliance defines a set of services which can satisfy the needs described above, exploiting Web Services technology. Having in mind the exploitation of cosmological surveys, we have implemented what we consider the most fundamental VO Web Services for our scientific interests: Conesearch (retrieves physical data values from a cone centered on one point in the sky - the simplest query), SkyNode (allows to filter on the physical quantities in the database in order to select a well defined data subset), SIAP (retrieves all the images contained in a sky region of interest), SSAP (retrieves 1D spectra). Our testing bench is the VVDSCDFS data set, made public in 2004, which contains photometric and spectroscopic information for 1599 sources (Le Fèrve et al., 2004, A\&A, 428, 1043, see <http://cencosw.oamp.fr>). On this data set, we have implemented and published on US NVO registry the first three services
\end{abstract}


mentioned above, to demonstrate the viability of this approach and its usefulness to the astronomical community. Implementation of SSAP service for spectra retrieval will be the next step.

\title{
The Golosiiv on-line plate archive database, management and maintenance
}

\author{
L. Pakuliak and T. Sergeeva \\ Department of Astrometry, Main Astronomical Observatory, \\ Zabolotnogo str.,27, Kiev, 03680 Ukraine \\ email: pakuliak,sergeeva@mao.kiev.ua
}

\begin{abstract}
We intend to create online version of the database of the MAO NASU plate archive as VO-compatible structures in accordance with principles, developed by the International Virtual Observatory Alliance in order to make them available for world astronomical community. The online version of the log-book database is constructed by means of MySQL+PHP. Data management system provides a user with user interface, gives a capability of detailed traditional form-filling radial search of plates, obtaining some auxiliary sampling, the listing of each collection and permits to browse the detail descriptions of collections. The administrative tool allows database administrator the data correction, enhancement with new data sets and control of the integrity and consistence of the database as a whole. The VO-compatible database is currently constructing under the demands and in the accordance with principles of international data archives and has to be strongly generalized in order to provide a possibility of data mining by means of standard interfaces and to be the best fitted to the demands of WFPDB Group for databases of the plate catalogues. On-going enhancements of database toward the WFPDB bring the problem of the verification of data to the forefront, as it demands the high degree of data reliability. The process of data verification is practically endless and inseparable from data management owing to a diversity of data errors nature, that means to a variety of ploys of their identification and fixing. The current status of MAO NASU glass archive forces the activity in both directions simultaneously: the enhancement of log-book database with new sets of observational data as well as generalized database creation and the cross-identification between them. The VO-compatible version of the database is supplying with digitized data of plates obtained with MicroTek ScanMaker 9800 XL TMA. The scanning procedure is not total but is conducted selectively in the frames of special projects.
\end{abstract}

\section{VObs.it - the Italian Virtual Observatory}

F. Pasian $^{1}$, U. Becciani ${ }^{2}$, S. Cassisi ${ }^{3}$, A. Fontana ${ }^{4}$, B. Garilli ${ }^{5}$, C. Gheller ${ }^{6}$, P. Giommi ${ }^{7}$, G. Longo ${ }^{8}$, A. Preite Martinez ${ }^{9}$, R. Smareglia ${ }^{1}$ and A. Volpicelli ${ }^{10}$

${ }^{1}$ INAF - O.A. Trieste, Via G. B.Tiepolo 11, I-34143 Trieste, Italy

${ }^{2}$ INAF - O.A. Catania, Via S. Sofia 78, I-95123 Catania, Italy

${ }^{3}$ INAF - O.A. Teramo, Via Mentore Maggini, I-64100 Teramo, Italy

${ }^{4}$ INAF - O.A. Roma, Via di Frascati 33, I-00040 Monte Porzio Catone, Italy

${ }^{5}$ INAF - IASF Milano, Via E. Bassini 15, I-20133 Milano, Italy

${ }^{6}$ CINECA, Via Magnanelli 6/3, I-40033 Casalecchio di Reno (Bologna), Italy

${ }^{7}$ ASI - Unità 'Osservazione dell'Universo', Viale Liegi 26, I-00198 Roma, Italy

${ }^{8}$ Università di Napoli 'Federico II', via Cinthia, I-80126 Napoli, Italy

${ }^{9}$ INAF - IASF Roma, Via del Fosso del Cavaliere 100, I-00133 Roma, Italy

${ }^{10}$ INAF - O.A. Torino, Via Osservatorio 20, I- 10025 Pino Torinese (TO), Italy email: pasian@oats.inaf.it

Abstract. The participation of Italy in VO activities has been initially delegated to a Grid project (i.e., DRACO). After the MoU between INAF and Euro-VO was signed, the VObs.it project was established. The aim of the VObs.it project is to coordinate within a unified approach the archives and databases developed by the Italian community. The first steps in this direction are to foster the adoption of IVOA standards, to provide Grid-aware VO 
applications and to build a national registry containing the list of VO-compliant services available to the international community. The Italian participation in EU-funded international projects is targeted to the development of tools to be subsequently used within the international VO. This allows our developers to be at the forefront of technology in the field. Equally important are participation in the IVOA Executive Committee and in the IVOA working groups defining standard protocols and formats. The activities forming the initial core of the VObs.it are the following: the Long-Term Archive of the TNG available at the INAF centre for Astronomical Archives (IA2), the archive of the VIMOS-VLT Deep Survey, DSS-II and GSC-II (both the databases and the all-sky uncompressed images), BaSTI a widely-used database of theoretical stellar evolution predictions specifically suited for population synthesis analysis, and the Italian Theoretical Virtual Observatory (ITVO) a distributed database of simulated data. Extension to other data and future projects (e.g., the raw LBT and science LBC data, VST) is foreseen. From an initial core of activities carried out by INAF, other groups and organizations are joining the collaboration and expanding VObs.it scientific and technological capabilities. CINECA is active in ITVO and in the definition of IVOA standards, while at the ASI Science Data Center VO-related work has started within the VObs.it and Euro-VO frameworks.

\title{
Interoperability and integration of theoretical data in the Virtual Observatory
}

\author{
F. Pasian $^{1}$, S. Ameglio ${ }^{2}$, U. Becciani ${ }^{3}$, S. Borgani ${ }^{2}$, C. Gheller ${ }^{4}$, V. Manna ${ }^{1}$, \\ P. Manzato ${ }^{1}$, L. Marseglia ${ }^{1}$, R. Smareglia ${ }^{1}$ and G. Taffoni ${ }^{1}$ \\ ${ }^{1}$ INAF - O.A. Trieste, Via G.B.Tiepolo 11, I- 34143 Trieste, Italy \\ ${ }^{2}$ Università di Trieste, Dip. Astonomia, Via G.B. Tiepolo 11, I-34143 Trieste, Italy \\ ${ }^{3}$ INAF - O.A. Catania, Via S.Sofia 78, I-95123 Catania, Italy \\ ${ }^{4}$ CINECA, Via Magnanelli 6/3, I-40033 Casalecchio di Reno (Bologna), Italy \\ email: pasian@oats.inaf.it
}

\begin{abstract}
The aim of the Virtual Observatory has recently expanded from seeking interoperability among astronomical catalogue and archive systems to including also access to analysis tools, computational services and numerical simulations. As a matter of fact, beside the observational data, there is also a huge amount of theoretical data generated by computer simulations that can be useful if published in Virtual Observatory compatible form. Therefore, considerable interest has been shown in including products of theoretical research. A data model for theoretical data is being designed. At the same time, an interim Simple Numerical Access Protocol (SNAP) as part of the Data Access Layer provides, through negotiation between the client and the theoretical dataset service, a standardized access mechanism to distributed theoretical data objects. We present the first integration within the Virtual Observatory of a set of theoretical data structured with a prototype of the SNAP data access protocol. Our resource provides not only access to simulation data stored in a dedicated archive but also, interfacing web services, a visualization service and the possibility to extract a number of astronomical observables. We focussed our work on a set of numerical simulations of galaxy clusters identified at redshift 0 and produced with the GADGET2 code. We show the possibility of computing the temperature and density profiles, of visualizing theoretical results with VO-enabled astronomical tools, of comparing the results with astronomical observations. The activity is being carried out as part of VO-Tech/DS4, ITVO and VObs.it projects.
\end{abstract}

\section{Hera - The HEASARC's new data analysis service \\ W.D. Pence and P. Chai \\ NASA Goddard Space Flight Center, Greenbelt, MD 20771, USA email: William.D.Pence@nasa.gov}

Abstract. Hera is the new data analysis service provided by the HEASARC at the NASA Goddard Space Flight Center that enables qualified student and professional astronomical researchers to immediately begin analyzing scientific data from astrophysics missions. All the 
necessary resources needed to do the data analysis are freely provided by Hera, including $(i)$ the latest version of the hundreds of scientific analysis programs all installed and ready to run; (ii) high-speed access to the terabytes of data in the HEASARC's data archive, (iii) a cluster of fast Linux workstations to run the software; and (iv) ample local disk space to temporarily store the data and results. Full information about how to use Hera is available from $<$ http://heasarc.gsfc.nasa.gov/hera $>$. Users only need to download the small Fv FITS file viewer program, which serves as the portal to the Hera services. Users can then access Hera either by using the Hera Graphical User Interface to control an interactive data analysis session on the Hera server machines, or by executing individual data analysis commands from a command window on the user's local machine. In the latter mode, any input data files on the user's local machine are automatically copied up to the Hera server machine where the software task is executed. Any output files from the task are then copied back to the user's machine. The Hera services have also been integrated into the HEASARC's data archive web pages, so researchers can simply click on a link to immediately begin analyzing the corresponding data set without having to first download the data or install any software other than Fv.

\title{
The Belgrade Plate Archive Database : current status and scientific tasks
}

\author{
V. Protitch-Benishek ${ }^{1}$, A. Mihajlov ${ }^{2}$, T. Jakshich ${ }^{3}$, and V. Benishek ${ }^{1}$ \\ ${ }^{1}$ Astronomical Observatory, Belgrade, Serbia \\ ${ }^{2}$ Faculty of Physics, Belgrade, Serbia \\ ${ }^{3}$ Department of Astronomy, Faculty of Mathematics, Belgrade, Serbia \\ email:vprotic@aob.bg.ac.yu
}

\begin{abstract}
The plate archives of the Belgrade Astronomical Observatory contain more than 15000 glass photographic plates from the period 1936-1996. In addition to the other equipment the Observatory disposed of four instruments devoted especially to astrophotographic observations: Zeiss Refractor 65/1055 cm with special camera, Zeiss Astrograph 16/80 cm, Zeiss Refractor $20 / 302 \mathrm{~cm}$ with two photographic cameras $16 / 80 \mathrm{~cm}$ and Askania Equatorial refractor $13.5 / 100 \mathrm{~cm}$. Scientific observations were performed in the framework of the programs like: minor planet follow-up, search for the new objects (33 new minor planets were discovered from BAO), comet investigation, systematic observations of the Sun, Moon, giant planets, natural and artifical satellites, variable stars, double and multiple stars, stellar clusters, etc. Rare phenomena, such as passages of Mercury and Venus across the solar disc, lunar occultations of stars and planets and special objects have been observed too. The current status of Belgrade Astrophtopgraphic Plate Archive (BAPA) Database is reported and a brief description of all phases of such a large Project is given. The preliminary computer-readable catalogue of relevant data from the period 1936-1966 is finished as a representative sample only. The Catalogue BAPA is included into WFPDB ( <http://www.skyarchive.org $>$ ) as one of the basic sources. A couple of statistical distributions as an example of the kind of informations which will be possible to extract from the database is given.
\end{abstract}

\section{Space-time coordinated metadata for the Virtual Observatory}

\section{A.H. Rots}

Harvard-Smithsonian Center for Astrophysics, 60 Garden Street, Cambridge, MA 02138, USA email: arots@head-cfa.harvard.edu

Abstract. Space-time coordinate metadata are at the very core of understanding astronomical data and information. This aspect of data description requires very careful consideration. The design needs to be sufficiently general that it can adequately represent the many coordinate systems and conventions that are in use in the community. On the other hand the most basic requirement is that the space-time metadata for queries, for resource descriptions, and for data be complete and self-consistent. It is important to keep in mind that space, time, redshift, and 
spectrum are strongly intertwined coordinates: time has little meaning without knowing the location, and vice-versa; redshift and spectral data require position and velocity for correct interpretation. The design of the metadata structure has been completed at this time and will support most, if not all, coordinate systems and transformations between them for the Virtual Observatory, either immediately or through extensions. This work has been supported by NASA under contract NAS 8-03060 to the Smithsonian Astrophysical Observatory for operation of the Chandra X-ray Center.

\title{
The Golosyiv plate archive digitisation
}

\author{
T.P. Sergeeva ${ }^{1}$, A.V. Sergeev ${ }^{2}$, L.K. Pakuliak ${ }^{1}$ and A.I. Yatsenko ${ }^{1}$ \\ ${ }^{1}$ Department of Astrometry, Main Astronomical Observatory, Kiev, 03680 Ukraine \\ ${ }^{2}$ Centre of Astronomical and Medico-Ecological Researches, Kiev, 03680 Ukraine \\ email: sergeev@mao.kiev.ua
}

\begin{abstract}
The plate archive of the Main Astronomical Observatory of the National Academy of Sciences of Ukraine (Golosyiv, Kyiv) includes about 85000 plates which have been taken in various observational projects during 1950-2005. Among them are about 25000 of direct northern sky area plates and more than 600000 plates containing stellar, planetary and active solar formations spectra. Direct plates have a limiting magnitude of 14.0-16.0 mag. Since 2002 we have been organising the storage, safeguarding, cataloguing and digitization of the plate archive. The very initial task was to create the automated system for detection of astronomical objects and phenomena, search of optical counterparts in the directions of gamma-ray bursts, research of long period, flare and other variable stars, search and rediscovery of asteroids, comets and other Solar System bodies to improve the elements of their orbits, informational support of CCD observations and space projects, etc. To provide higher efficiency of this work we have prepared computer readable catalogues and database for 250000 direct wide field plates. Now the catalogues have been adapted to Wide Field Plate Database (WFPDB) format and integrated into this world database. The next step will be adaptation of our catalogues, database and images to standards of the IVOA. Some magnitude and positional accuracy estimations for Golosyiv archive plates have been done. The photometric characteristics of the images of NGC 6913 cluster stars on two plates of the Golosyiv's double wide angle astrograph have been determined. Very good conformity of the photometric characteristics obtained with external accuracies of 0.13 and $0.15 \mathrm{mag}$. has been found. The investigation of positional accuracy have been made with A3 \pm format fixed bed scanner (Microtek ScanMaker 9800XL TMA). It shows that the scanner has non-detectable systematic errors on the X-axis, and errors of $\pm 15 \mu \mathrm{m}$ on the Y-axis. The final positional errors are about $\pm 2 \mu \mathrm{m}\left( \pm 0^{\prime \prime} .2\right)$. have been obtained after corrections for systematic errors of the scanner and averaging four scans. So we may conclude that astrometric and photometric investigations may be done with precise commercial scanners. It will be necessary to scan plates at a minimum of two positions. We plan to scan the plate archive according to the priority of scientific tasks. Scanning will be done with an optical resolution of $1200 \times 1200 \mathrm{dpi}$ (pixel size $20 \mu \mathrm{m}$ ), and with maximum amplitude resolution. The plate archive of MAO NASU is a unique well equipped instrument for conducting a range of astronomical investigations with a time scale of more than $50 \mathrm{yr}$.
\end{abstract}

\section{VOSED: a tool for the characterization of developing planetary systems}

\author{
E. Solano ${ }^{1}$, R. Gutiérrez ${ }^{1}$, A. Delgado ${ }^{1}$, L.M. Sarro ${ }^{2}$ and B. Merín ${ }^{3}$ \\ ${ }^{1}$ SVO-LAEFF. P.O. Box 50727, E-28080 Madrid, Spain \\ ${ }^{2}$ SVO-UNED. Dpto. Inteligencia Artificial, C/ Juan del Rosal 16, E-28040 Madrid, Spain \\ ${ }^{3}$ Leiden Observatory, P.O. Box 9513, NL-2300RA, Leiden, the Netherlands \\ email: esm,raul,Arancha.Delgado@laeff.inta.es, lsb@dia.uned.es, merin@strw.leidenuniv.nl
}

Abstract. The transition phase from optically thick disks around young pre-main sequence stars to optically thin debris disks around Vega type stars is not well understood and plays 
an important role in the theory of planet formation. One of the most promising methods to characterize this process is the fitting of the observed SED with disk models. However, despite its potential, this technique is affected by two major problems if a non-VO methodology is used: on the one hand, SEDs building requires accessing to a variety of astronomical services which provide, in most of the cases, heterogeneous information. On the other hand, model fitting demands a tremendous amount of work and time which makes it very inefficient even for a modest dataset. This is an important issue considering the large volume of data that missions like Spitzer is producing. In the framework of the Spanish Virtual Observatory (SVO) we have developed VOSED < http://sdc.laeff.inta.es/vosed/> an application that permits to characterize the protoplanetary disks around young stars taking advantage of the already existing VO standards and tools. The application allows the user to gather photometric and spectroscopic information from a number of VO services, trace the SED, and fit the photospheric contribution with a stellar model and the IR excess with a disk model. The Kurucz models described in Castelli et al. (1997, A\&A, 318, 841) are used to reproduce the photospheric contribution whereas the grid of models of accretion disks irradiated by their central stars developed by D'Alessio et al. (2005, $<$ http://cfa-www.harvard.edu/youngstars/dalessio $>$ ) is used for the disk contribution. In both cases, the models are retrieved from the SVO Theoretical Model Web Server using the TSAP protocol. As pointed out before, model fitting constitutes a fundamental step in the analysis process. VOSED includes a tool to estimate the model parameters (both stellar and disk) based on bayesian inference. The main aim of the tool is to quantitatively analyse the data in terms of the evidence of models of different complexity, evaluate what other alternative models can compete with the most a posteriori probable one and what are the most discriminant observations to discard alternatives.

\title{
The 2XMM pre-release catalogue: a test case for VO cross correlation of large archives
}

\author{
J.A. Tedds ${ }^{1}$, D. Law-Green ${ }^{1}$, M.G. Watson ${ }^{1}$, K.T. Noddle ${ }^{1}$, D. Morris ${ }^{2}$ \\ and N.A. Walton ${ }^{2}$ \\ ${ }^{1}$ Dept. of Physics 85 Astronomy, University of Leicester, Leicester LE1 7RH, UK \\ email: jat@star.le.ac.uk \\ ${ }^{2}$ Institute of Astronomy, Madingley Road, Cambridge CB3 OHA, UK
}

\begin{abstract}
The XMM-Newton Survey Science Centre has made a pre-release of the second serendipitous source catalogue, 2XMM. This is the largest X-ray source catalogue ever made, including $\sim 150 \mathrm{~K}$ objects derived from $\sim 2500$ XMM-Newton observations since launch and covering a total sky area of approximately 400 sq.deg. The typical flux limit of the survey is $\sim 10^{-14} \mathrm{cgs}$, well matched to the dominant source population of the cosmic X-ray background emission. 2XMM will be a unique database to carry out evolutionary studies of different X-ray source populations. In order to maximise the scientific potential of $2 \mathrm{XMM}$ it is crucial to cross correlate with multi-wavelength archives and hence characterise the various source samples in detail. One can then go on to identify rare and unique sources as well as addressing crucial science questions using the large, statistically significant samples identified. These serve as the essential training sets for statistical identifications and VO data mining tools. We describe the work undertaken to make the catalogue fully VO compliant via the LEDAS high energy database and identify strategies to address the technical challenges of cross correlating a large catalogue such as this with other prime archives such as SDSS DR4 and UKIDSS. We will illustrate how 2XMM is being used as a test case within the VO community by the UK AstroGrid project to address the complex requirements of astronomers. This includes the handling of multiple matches to other catalogues and the adoption of a figure of merit to grade quality of matches that is not based on variable radius with error alone but can assign probabilities based on sky density and quality criteria from within each archive.
\end{abstract}




\title{
A VO 'container' for astronomical optical/UV spectra
}

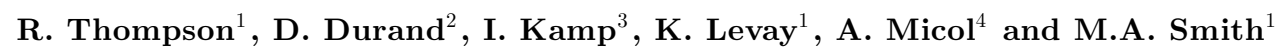 \\ ${ }^{1}$ STSCI/CSC, Baltimore, USA \\ ${ }^{2} C A D C$, Victoria, Canada \\ ${ }^{3}$ ESA/STSCI, Baltimore, USA \\ ${ }^{4}$ ESA/ST-ECF, Garching, Germany \\ email: msmith@stsci.edu
}

\begin{abstract}
FITS formats have provided a convenient means to access and interpret spectroscopic data for many years. However, each mission has been free to choose its FITS flavor and provide necessarily instrument specific keywords during this time. Over time, and with new missions developed with unique instrumental configurations, this has created a challenge for spectroscopic applications, which must be written in a complex fashion to recognize, read, and decipher the idiosyncrasies relating to each instrument. Moreover, some HST heritage instruments (GHRS, FOS) have stored different vectors in separate files, requiring their assembly before they can be used. With the advent of the VO and the first generation Simple Spectral Access Protocol (SSAP), it is possible to design a Spectral Container that addresses these issues by serving as a translation layer between the standardized VO protocols and the current FITS file formats. We have constructed SSAP services that point to a secondary data archive holding Spectral Container-packaged files for several MAST (Multi-Mission Archive at Space Telescope) missions. To date, these missions include those for which single-order observations are available: GHRS, FOS, EUVE, HUT, WUPPE, IUE, and STIS. In this poster we discuss the current status and examples of the Container using the Specview and VOSpec applications. We also discuss the need for second-generation VO protocols that will provide for multiple spectra (echelle multi-orders, time-series) within a single Container file.
\end{abstract}

\section{An alternative catalogue collection and a system to exploit it: CATS}

O. Verkhodanov ${ }^{1}$, S. Trushkin ${ }^{1}$, H. Andernach ${ }^{2}$ and N. Chernenkov ${ }^{1}$

${ }^{1}$ Special Astrophysical Observatory, RAS, 369167 Karachaevo-Cherkesia, Russian Federation

${ }^{2}$ Departamento de Astronomía, Universidad de Guanajuato, Guanajuato CP 36000, Mexico email:vo@sao.ru

Abstract. Conceived in 1996 to support observations with the RATAN-600 radio telescope, CATS is now a publicly accessible database allowing to search through $\sim 400$ catalogs, all of them accessible by anonymous $\mathrm{ftp}$, with a total of $\sim 109$ records $(<\mathrm{http}: / /$ cats.sao.ru $>$ ). CATS' content overlaps only partly with that of VizieR at CDS and is more complete for radio sources due to contributions from the catalog collection of one of us (HA, see Norris et al., this volume, for a summary on SpS6). Similar to existing databases CATS offers data extraction both interactively and via batch requests to jcats@sao.ru>, with object selection either from a subset or from all available catalogues, by coordinates, flux, frequency, and other parameters. CATS offers a larger variety of radio source lists but only partial inter-catalog cross-identifications. It also provides interactive creation of radio spectra of certain source samples as well as over 1300 radio images of Galactic SNRs.

\section{AstroGrid Virtual Observatory release 2006.3}

\author{
N.A. Walton and the Astrogrid Consortium \\ Institute of Astronomy, University of Cambridge, Madingley Road, Cambridge, CB30HA, UK \\ email: naw@ast.cam.ac.uk
}

Abstract. AstroGrid (<http://www.astrogrid.org $>$ ), the UK's Virtual Observatory system, will be releasing its latest fully operational release system, 2006.3, prior to the IAU XXVI General Assembly. This poster describes the increased functionalities offered to the end user in the 2006.3 release. The key data resources and applications accessible through the AstroGrid workbench will be itemised, Details of how astronomers can gain access to the system will be given. We 
note the use of technologies and interoperability standards, and how use of AstroGrid gives end user access truly global resources, including large repositories of data across the UK, Europe, the USA and elsewhere. We show how the astronomer can easily discover and visualise data from any major data centre by use of the 'AstroScope'. How then data from this can be stored in 'MySpace' and processed using applications either on the uses local machine or on remote servers. Specific example science use cases are noted, ranging from the mining of deep field survey data to investigate the clustering of galaxies at high redshifts, to studies of active regions on our Sun. AstroGrid is ready to try out, and to use - just go to <http://www.astrogrid.org/launch> to find out how. Without registration you'll be able to use AstroScope to discover and locally visualise data. With a simple registration procedure you'll gain access to the full features of the AstroGrid Workbench - including being able to save your data and results to your MySpace virtual storage. See $<$ http://software.astrogrid.org $>$ for further details. 